\title{
Le burin des Vachons : apports d'une relecture technologique à la compréhension de l'Aurignacien récent du nord de l'Aquitaine et des Charentes
}

Burin des Vachons: a technological reconstruction approach to understanding the recent Aurignacian in Northern Aquitaine and Charente

\section{Damien Pesesse et Alexandre Michel}

\section{OpenEdition}

Journals

Édition électronique

URL : http://journals.openedition.org/paleo/184

DOI : $10.4000 /$ paleo. 184

ISSN : 2101-0420

Éditeur

SAMRA

Édition imprimée

Date de publication : 1 décembre 2006

Pagination : 143-160

ISSN : 1145-3370

Référence électronique

Damien Pesesse et Alexandre Michel, «Le burin des Vachons : apports d'une relecture technologique à la compréhension de l'Aurignacien récent du nord de l'Aquitaine et des Charentes », PALEO [En ligne], 18 | 2006, mis en ligne le 23 avril 2009, consulté le 07 juillet 2020. URL : http:// journals.openedition.org/paleo/184 ; DOI : https://doi.org/10.4000/paleo.184

\section{cc) (†)}

PALEO est mis à disposition selon les termes de la licence Creative Commons Attribution - Pas d'Utilisation Commerciale - Pas de Modification 4.0 International. 


\title{
LE BURIN DES VACHONS : APPORTS D'UNE RELECTURE TECHNOLOGIQUE À LA COMPRÉHENSION DE L'AURIGNACIEN RÉCENT DU NORD DE L'AQUITAINE ET DES CHARENTES
}

\author{
Damien PESESSE ${ }^{(1)}$ et Alexandre MICHEL ${ }^{(2)}$
}

\begin{abstract}
Résumé : La couche 2 des Vachons a livré un type de burin particulier reconnu par J. Coiffard (Coiffard 1914, 1922), puis J. Bouyssonie (Bouyssonie 1948) et défini par M. Perpère comme le " burin des Vachons " (Perpère 1972 a et b). Le façonnage de ces burins correspond à une intention de production lamellaire orientée vers la recherche de lamelles à tendance rectiligne, mesurant de 20 à $60 \mathrm{~mm}$ de long. Un schéma opératoire complexe peut alors être restitué. Cette redéfinition confirme la forte charge culturelle de ce procédé, dont témoigne sa position chronologique dans les gisements du nord de l'Aquitaine.

En effet, les ensembles comprenant des burins des Vachons clôturent certaines séquences aurignaciennes classiques dont la Ferrassie, le Roc-de-Combe, l'Abri Pataud, le Facteur et le Flageolet I. La nature du procédé opératoire et de l'objectif sous-jacent révèlent un changement fort dans les conceptions lamellaires de la fin de l'Aurignacien, en décalage avec les productions antérieures issues des burins busqués. Ce travail propose de documenter cette évolution technique qui permet d'envisager sur des bases nouvelles les demières phases de l'Aurignacien.
\end{abstract}

Mots-clés : Aurignacien récent, burin des Vachons, technologie lithique, production lamellaire, Aquitaine.

Key-words: Recent Aurignacian, burin des Vachons, lithic technology, lamellar production, Aquitaine.

\section{Abridged english version \\ BURIN DES VACHONS: A TECHNOLOGICAL RECONSTRUCTION APPROACH TO UNDERSTANDING THE RECENT AURIGNACIAN IN NORTHERN AQUITAINE AND CHARENTE}

The Vachons (located in Voulgézac, Charentes, north Aquitain, France) correspond to diffe rent rock-shelters and caves. The site has been explored by $A$. Trémeau de Rochebrune in 1867 and by G. Chauvet in 1896. Main excavations have been realised by J. Coiffard at the end of the XIX ${ }^{\circ}$ century (Coiffard 1914,1922), and by J. Bouyssonie from 1929 to 1937 (Bouyssonie et Sonneville-Bordes 1956). They excavated two rock-shelters, called Abri 1 and Abri 2, and one cave. In the shelters, a very similar stratigraphy has been recognized, starting with a classical Aurignacian, through a recent Aurignacian, followed by three gravettian levels. The cave stratigraphy is mo re recent, starting with the Gravettian to finish with the Solutrean (Bouyssonie 1948).

$J$. Coiffard and J. Bouyssonie have very soon recognized the specificity of the second level of both shelters corresponding to a recent aurignacian. These levels are characterized by the predominance of specific burins. In 1972, M. Perpère formulated the first definition of those burins, named " burins des Vachons". They are distinguished after the importance of the spall removals, their constant lateralisation, and the presence of flat removals (Perpère $1972 \mathrm{a}$ and b). Since, numerous burins presenting flat spalls removal have been called "burins des Vachons", with no consideration for the chrono-cultural context in which those pieces have been found. A new analysis of the burin des Vachons, coming from Coiffard's excavations in the 2nd rock-shelter of the Vachons lead to a new interpretation of this tool. A technological approach argues that the shaping of this tool corresponds indeed to a lamellar production process. In this acceptation, the Vachons recovers a strong chrono-cultural value as discussed below.

Burins des Vachons are realised on diffe rent kinds of blanks, including blades, small blocs, and a large part of thick cortical flakes. Those morphological variations are correletad to distinct preparations of the blanks. Because of their regularity, blades do not need a very important shaping, but irregular or cortical flakes do need a preparation which can be almost complete. Some cores are also entirely shaped through a peripheric crest, which is original for aurignacian lithic productions. This shaping results in a straight morphology, in which the production will be conducted. Considering the thickness of the core, spall removals are more or less extracted on the flanks. The goal is to define the first intention product in the center of the debitage surface, defined by lateral removals.

By the time Coiffard performed his excavations, bladelets and small fragments resulting from the shaping were missed and therefore absent of his collection. Then the morphology of the sought bladelets can be infered through the observation of the spall removal nega-

(1) Damien PESESSE, ESEP - UMR 6636, Maison Méditerranéene des Sciences de l'Homme, 5 rue du château de l'Horloge, BP 647, 13094 Aix-en-Provence Cedex 2

(2) Alexandre MICHEL, Université Bordeaux 1, PACEA-IPGQ - UMR 5199, Avenue des Facultés, 33405 Talence Cedex 
tives and the comprehension of the process logic. Bladelets produced are straight and rectilinear, and show a sharp extremity, due to the basal convergence of the removals. The dimensions observed on the cores are 17 to $80 \mathrm{~mm}$ long, for 3 to $12 \mathrm{~mm}$ wide. The burin des Vachons can then be considered as the result of a very specific lamellar process, and not only as a flat burin.

According to this definition, the burin des Vachons recovers a specific chrono-stratigraphical position. Indeed, archeological levels including this object are Abri du Facteur, layers 17 and 15 (Delporte 1962, 1968), Roc de Combe, layers 6 and 5 (Demars et Laurent 1989 ; Demars 1994), Flageolet I, layer 8 (Rigaud 1982), Ferrassie, levels K3b and uppers (Delporte 1984), Abri Pataud, layers 7 and 6 (Chiotti 2003). All these levels share one principal characteristic : they are the latest manifestations of classic aurignacian archeological sequences in the Northem Aquitaine. It stresses the fact that burin des Vachons is a very good indicator of the latest technologies employed by Aurignacians (Demars 1994 ; Demars et Laurent 1989). In those layers, a kind of straight and rectilinear bladelet with direct marginal retouch exists (Rigaud 1982, Delporte 1984), which is very close to the lamellar negatives observed on the Vachons. Those bladelets may result from this process, but refittings are neccessary to confirm this hypothesis.

This assertion makes sense in comparison to the lamellar process traditionnaly related to the recent aurignacian : the burin busqué lamellar process. This technology produces very small and normalized twisted bladelets, with inverse retouch, i.e. Dufour bladelet, subtype Roc-de-Combe (Demars et Laurent 1989, Lucas 1997, 2000 ; Chiotti 1999, 2003; Bordes 2005 ; Bordes et Lenoble 2002 ). Burin des Vachons and burin busqué represent two opposite technical process. But burin busqué produces also a specific retouched bladelet, the Caminade bladelet (Bordes et Lenoble 2002), which might share technical characteristics with the blank coming from the Vachons's production.

So, even if the technical evolution from the burin busque to the burin des Vachons is not yet formalized, a deep modification of the lamellar design at the end of the Aurignacian can be observed. Those mutations are the symptom of changes occuring in aurignacian societies. The substitution of the burin busqué technology, which is conceptually a very rigid process, by the burin des Vachons foreshadows, in a more adequate way, the future technical evolutions at that time.

\section{INTRODUCTION}

La première moitié du XXème siècle a vu émerger les principales appellations d'outils, encore usitées aujourd'hui. Dans cette phase de désignation et de caractérisation des industries, le nom du site éponyme a souvent été employé pour singulariser un outil, définissant alors un type particulier. Ces termes eurent des avenirs contrastés, certains furent adoptés très rapidement, d'autres concurrencés, puis remplacés, voire oubliés (Le Brun-Ricalens 2005). À ces péripéties historiographiques, s'ajoute parfois un glissement terminologique. Ainsi, un décalage peut également se dessiner entre le sens initial d'une appellation et l'acception qui en est faite quelques décennies plus tard (Brézillon 1968 ; Le Brun-Ricalens 2005).

L' " histoire " du burin des Vachons illustre bien le long processus qui se déroule entre la découverte d'un outil, la reconnaissance de ses spécificités et les différentes phases de description. Le burin des Vachons, mis au jour à la fin du XIXème siècle par J. Coiffard, identifié par J. Bouyssonie en 1948, puis défini par M. Perpère en 1972, se trouve actuellement dans sa phase de caractérisation technologique.

La dénomination "Vachons " a été appliquée à de nombreux burins carénés plans dont les enlèvements tendent à envahir la face inférieure de l'éclat, conférant à ce " type " une définition très large et, par conséquent, assez floue. Un retour à la série éponyme, objet de cet article, révèle clairement que l'appellation Vachons ne peut se résumer ainsi, et renvoie au contraire à une réalité technique bien plus spécifique, qu'il paraît pertinent de préciser et définir. Une fois redéfini et replacé dans son contexte chronologique, le burin des Vachons illustre bien plus qu'une anecdote technologique : il semble synonyme d'un changement fort dans les conceptions et les objectifs de la production lamellaire qui accompagne les demières phases de l'Aurignacien.

\section{LA STATION DES VACHONS}

La station des Vachons, commune de Voulgézac, Charente, correspond à une suite d'abris et de grottes, s'ouvrant sur la partie méridionale d'un vallon dans lequel serpente la biennommée Font-Robert. Dès 1867, A. Trémeau de Rochebrune, puis G. Chauvet en 1896, commencèrent les premières prospections et ramassages. Peu après J. Coiffard entreprit des fouilles (Coiffard 1914,1922) dans l'Abri 1 et plus particulièrement dans l'Abri 2. P. David réalisa ensuite une tranchée dans la grotte attenante. De 1929 à 1937, les fouilles furent menées de concert par J. Coiffard et J. Bouyssonie (Bouyssonie et Sonneville-Bordes 1956 ; Fontaine 2000). Le chanoine de Brive effectua dans l'Abri 1 une tranchée perpendiculaire à la cavité, puis prolongea la fouille de P. David dans la grotte de l'Oeil-de-boeuf.

\section{Archéo-séquence}

J. Bouyssonie reconnut cinq niveaux archéologiques dans l'Abri 1, allant de l'Aurignacien au Gravettien final, et un seul niveau gravettien sur la plateforme inférieure du même abri (Bouyssonie 1948). L'Abri 2 livra à J. Coiffard une séquence similaire (Bouyssonie et Sonneville-Bordes 1956). La séquence de la grotte est légèrement plus récente. Dans cette cavité, les quatre ensembles rencontrés débutent au Gravettien pour s'achever avec le Solutréen. La qualité de la lecture stratigraphique des fouilleurs permet à partir de leurs observations de restituer avec plus de précision la séquence archéologique.

Dans l'Abri 1, à la base du niveau 1, J. Bouyssonie mentionne la présence " d'un lit assez mince et poussiéreux, mais gris" contenant des pièces aurignaciennes, mais aussi " des éclats de facture moustérienne, voire un biface" (Bouyssonie 1948, p. 5). Cette observation fut reprise dans la publication suivante: "il est vraisemblable de supposer qu'une trace de niveau moustérien existait aussi à 


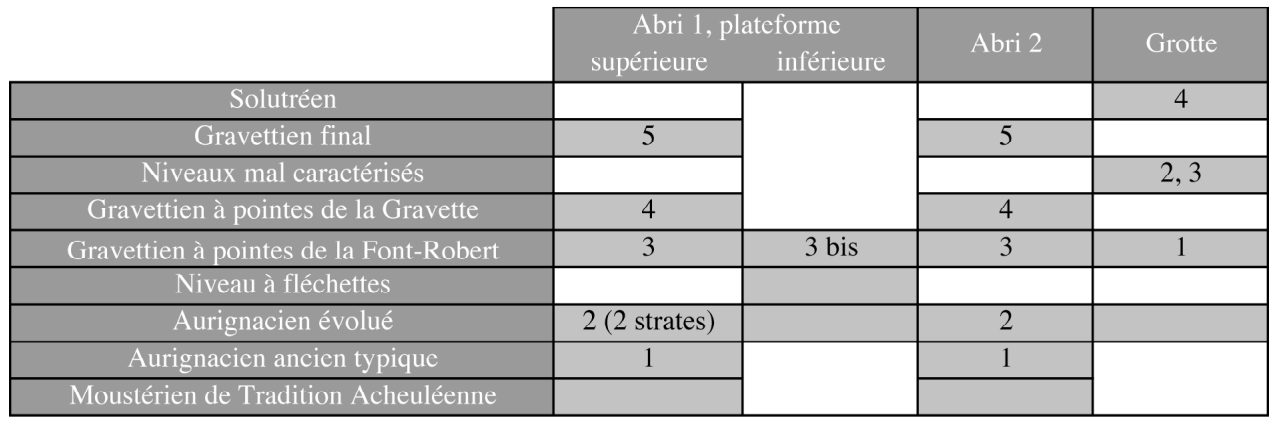

Tableau 1-

Archéo-stratigraphie de la Station des Vachons.

Table 1-

Vachons's station

archeo-stratigraphy.
l'Abri $n^{\circ} 2$ " (Bouyssonie et Sonneville-Bordes 1956, p. 279). En effet, de nombreux éléments lithiques peuvent être rapportés à une occupation du Paléolithique moyen : racloirs, denticulés, supports issus de productions discoïdes, biface et fragment de biface, tous deux de type MTA (comm. pers. J.-Ph. Faivre).

Selon J. Bouyssonie, la couche 2 de l'Abri 1 correspond peut-être à " deux strates, assez friables en arrière, très compactes en avant, où elles se trouvent coincées sous des blocs tombés " (Bouyssonie 1948, p. 6). Enfin, si un seul ensemble est individualisé sur la plateforme inférieure de l'Abri 1, le chanoine identifia " plusieurs lits minces noirâtres, sensiblement horizontaux, intercalés de lits sableux " (Bouyssonie 1948, p. 8). II note que "les plus inférieurs de ces foyers bousculés contenaient pas mal de grattoirs carénés et de rabots [et] pourraient être contemporains de la couche 2 de l'Abri 1. À peu près au même niveau, j'ai remarqué de jolis spécimens de "fléchettes " du type de La Gravette; les autres foyers, plus ou moins superposés, fournirent toujours des pointes à pédoncule " (Bouyssonie, 1948, p. 9). Ces éléments témoignent en faveur de l'existence de plusieurs niveaux archéologiques identifiés lors de la fouille, mais non isolés les uns des autres.

Enfin, dans la grotte, J. Bouyssonie mentionne la présence d'un niveau d'Aurignacien évolué, comparable à celui de la couche 2, situé un peu en avant (Bouyssonie 1948, p. 35). Ces observations permettent d'affiner la stratigraphie habituellement retenue, bien que cela ne corresponde vraisemblablement qu'à une vague image de ce que fût la séquence archéologique complète (tab. 1).

\section{La couche 2 des Abris 1 et 2 des Vachons}

Les burins des Vachons furent rencontrés dans des ensembles comparables dans les quatre locus fouillés (Bouyssonie 1948 ; Bouyssonie et Sonneville-Bordes 1956 ; Perpère 1972 a et $b, 1977)$. Sa position stratigraphique peut être définie comme sus-jacente à l'Aurignacien typique dans l'Abri 1, sous-jacent au Gravettien ancien devant ce même abri, dans le prolongement horizontal de l'Aurignacien typique de l'Abri 2, et pour la grotte, sur le substrat, en avant, apparemment déconnecté de la séquence intérieure.

La collection des Vachons fut dispersée dans divers musées et institutions dont le Centre Régional d'Archéologie de Poitiers, I'Institut de Paléontologie Humaine (Fontaine 2000) et le musée Henri Barré de Thouars dans les Deux-Sèvres.
La série de la couche 2 abordée ici provient des fouilles réalisées par J. Coiffard. II en fit don, en 1900, au Musée des Eyzies, actuel Musée National de Préhistoire ; elle provient majoritairement de l'Abri 2 (tabl. 2). Son intégrité peut être largement remise en question par la présence d'éléments lithiques attribuables à différentes périodes du Paléolithique : éléments moustériens cités précédemment, puis un outillage comparable à celui de la couche 1 , dont de nombreux grattoirs carénés et une lame étranglée, et enfin, une microgravette proche de celles de la couche 3 .

\begin{tabular}{|c|c|c|}
\hline & Abri 1 & Abri 2 \\
\hline \multicolumn{3}{|l|}{ Nucléus } \\
\hline lames & 1 & \\
\hline éclats & 2 & 9 \\
\hline \multicolumn{3}{|l|}{ Lames } \\
\hline brute & 1 & 1 \\
\hline grattoir & 1 & 7 \\
\hline appointée & 1 & 1 \\
\hline burin & 5 & 4 \\
\hline retouchée & 2 & 7 \\
\hline étranglée & 1 & \\
\hline pièce esquillée & 1 & 3 \\
\hline \multicolumn{3}{|l|}{ Lamelles } \\
\hline grattoir caréné & 8 & 4 \\
\hline grattoir à museau & 11 & \\
\hline burin busqué & 5 & 8 \\
\hline burin busqué atypique & 3 & 5 \\
\hline burin des Vachons & 16 & 31 \\
\hline autre & 5 & 5 \\
\hline éléments lamellaires & 2 & \\
\hline \multicolumn{3}{|l|}{ Eclats } \\
\hline brut & & 7 \\
\hline retouché & 15 & 47 \\
\hline Biface & 1 & \\
\hline Total & 81 & 139 \\
\hline
\end{tabular}

Tableau 2 - Description typologique de l'assemblage de la couche 2 des Vachons.

Table 2 - Typological description of the Vachons layer 2. 

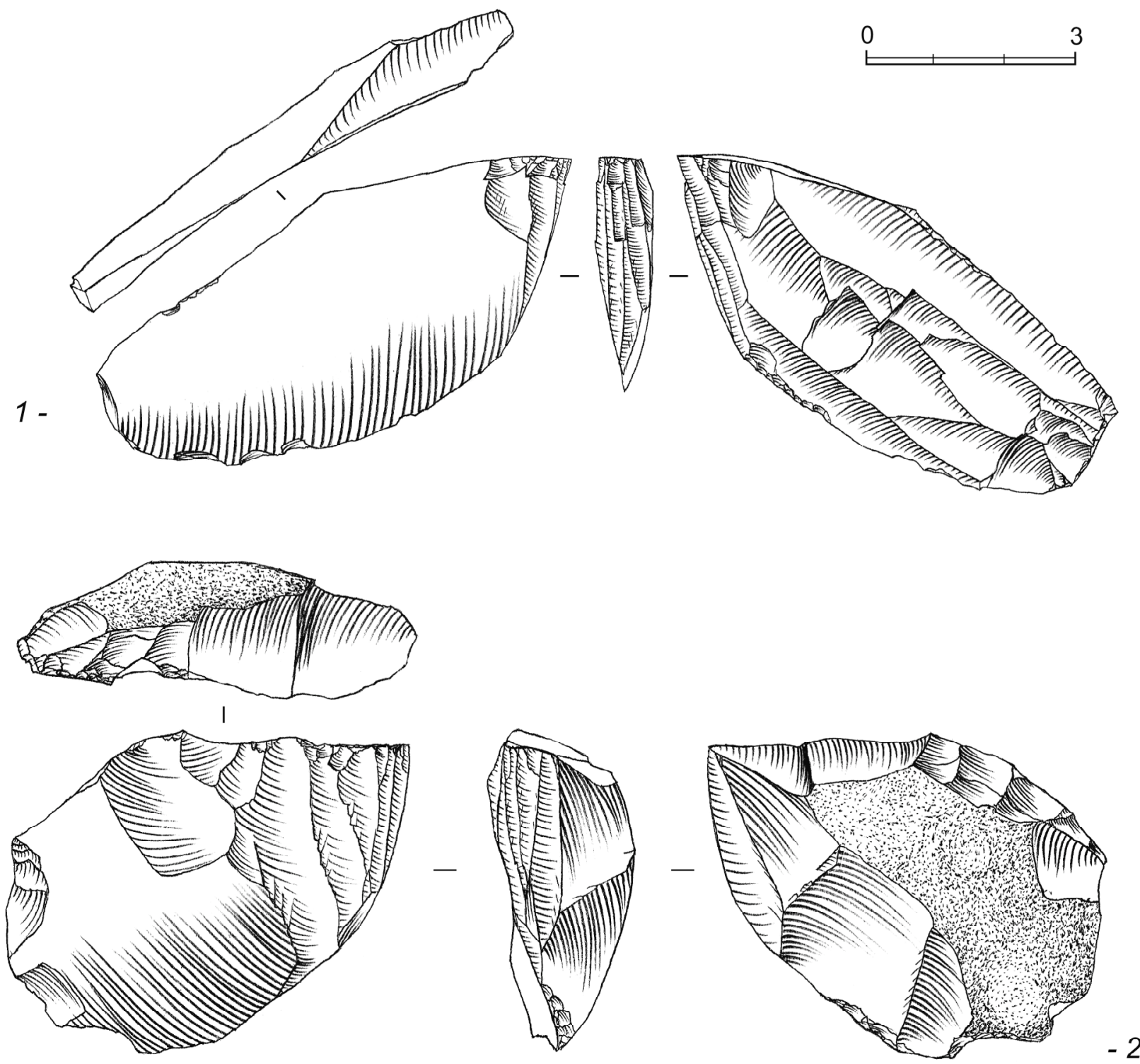

Figure 1 - Burins des Vachons, Abri 2, couche 2 (dessins D. Pesesse).

Figure 1 - Burins des Vachons, shelter 2, layer 2 (drawings D. Pesesse).

Compte tenu de ces biais, le mobilier présent ne peut être abordé comme un système technique cohérent et donc être interprété de manière globale. C'est donc à une approche du burin des Vachons partiellement déconnecté de son contexte industriel que nous contraint cet assemblage.

\section{DÉFINITIONS}

Très tôt l'abbé J. Bouyssonie distingua les burins rencontrés à l'Abri des Vachons: "Ici, nos ouvriers sont arrivés à une perfection remarquable : en amincissant la pièce par des retouches latérales en écaille, ils obtiennent ce qu'on pounait appeler un burin caréné plan ou pointu" (Bouyssonie 1948, p. 16). En 1956, il affine leur caractéri- sation : ces burins "ont en commun le fait qu'à un enlèvement unique, celui qui est parallèle à l'axe de la pièce, est opposé un groupe d'enlèvements étroits obtenus par une technique lamellaire identique à celle employée pour les grattoirs carénés les moins larges " (Bouyssonie et Sonneville-Bordes 1956, p. 284). Toutefois, ce burin ne fut pas introduit dans la liste typologique du Paléolithique supérieur (Sonneville-Bordes et Perrot 1956), ni dans celle révisée en 1972 (non publiée), mais intégré au manuel de typologie de Heinzelin (Heinzelin 1962, p. 30). M. Brézillon compara ensuite ces pièces à des " burins carénés sans encoche " (Brézillon 1968, p. 181). En 1972, M. Perpère individualisa enfin ces outils sous le terme " burin des Vachons" (Perpère 1972a, p. 414 ; 1972b, p. 321). Les critères de définition retenus concernent la présence d'un 

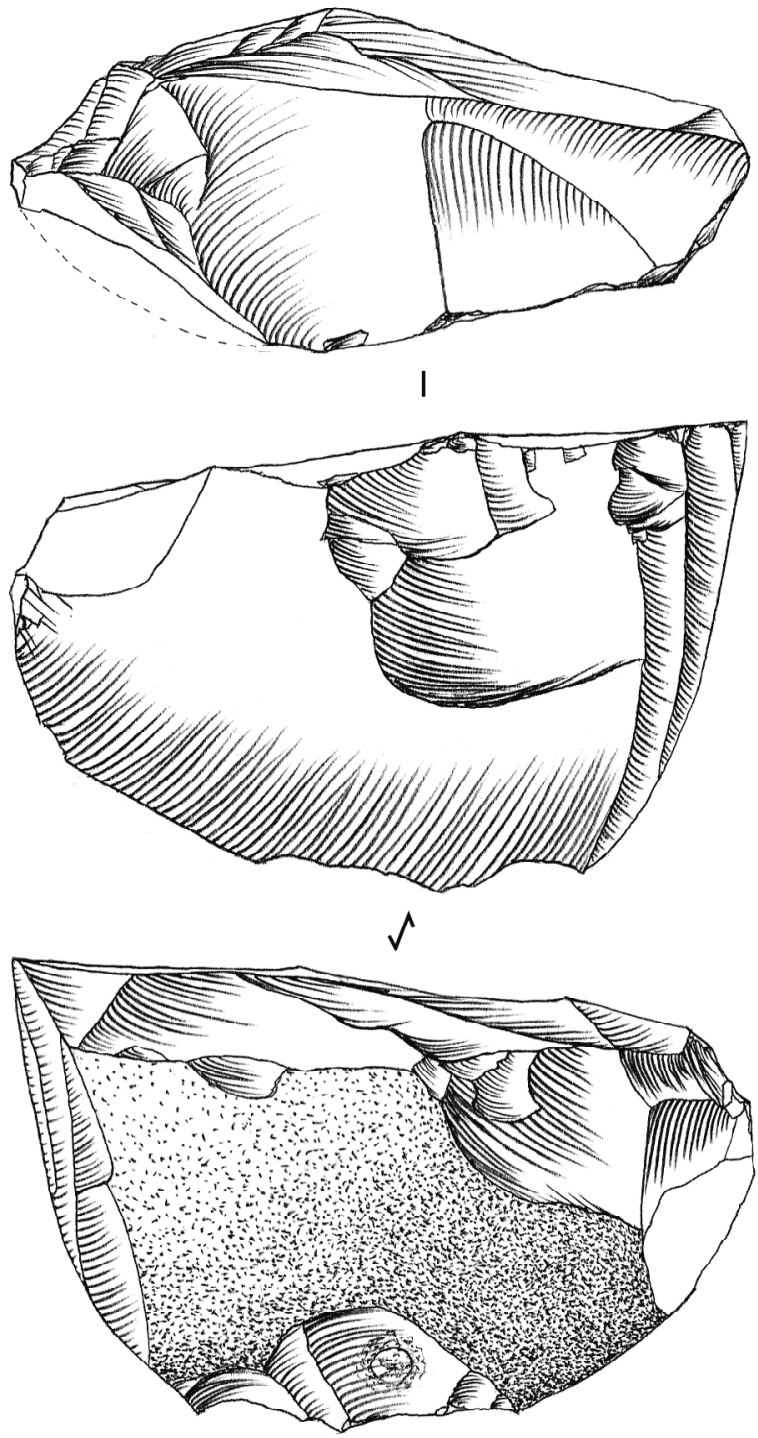

pan unique préférentiellement à droite, des enlèvements plans multiples à gauche, une retouche inverse du bord droit prolongeant ces négatifs, la présence d'une coche sur le bord gauche et l'implantation du burin fréquemment sur la partie proximale d'un éclat cortical épais (Perpère 1972a, p. 416). Cette définition fut reprise dans le demier essai de classification des outils du Paléolithique supérieur (Demars et Laurent 1989).

\section{Le burin des Vachons}

Le burin des Vachons constitue une catégorie apparemment polymorphe. Les aménagements varient en effet de manière très prononcée. Toutefois, la mise en forme de ces pièces conduit toujours à interpréter ces aménagements comme tributaires d'une intention de production lamellaire, ce que la restitution des logiques opératoires semble confirmer.
Figure 2 - Burins des Vachons, Abri 2, couche 2 (dessins D. Pesesse).

Figure 2 - Burins des Vachons, shelter 2, layer 2 (drawings D. Pesesse).

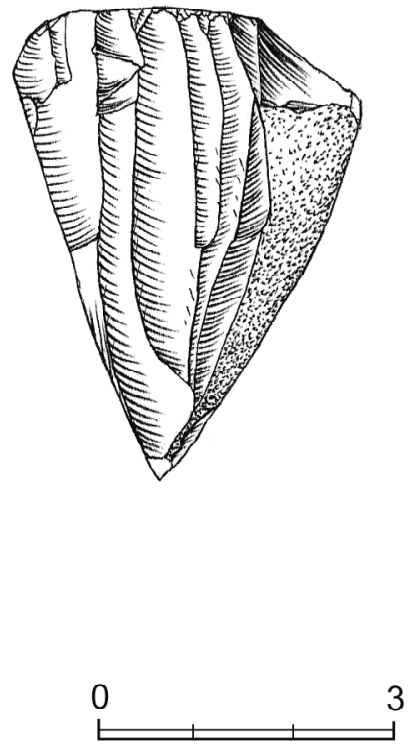

Ces burins sont réalisés sur des supports de natures diverses, comprenant des lames, des sous-produits du débitage laminaire, et une fraction importante d'éclats, fréquemment corticaux. Les supports recherchés sont souvent asymétriques et présentent parfois un dos naturel qui sera utilisé comme plan de frappe, brut ou après une modification du bord par une retouche directe abrupte.

La variabilité morphologique initiale des supports est corrélée à une mise en forme différentiée, sensiblement liée à la régularité et à l'épaisseur du support transformé. Les supports les plus fins, comme les lames, ne sont ainsi pratiquement pas investis (fig. 1, $n^{\circ} 1$ ). A l'opposé, l'exploitation de supports épais, notamment des éclats corticaux, induit un aménagement accru (fig. $1, \mathrm{n}^{\circ} 2$; fig. 2), voire complet, de la face inférieure (fig. 3). Les exemplaires les plus investis témoignent de mises en forme complètes, par le biais d'une crête périphérique, structurant totalement le 
0

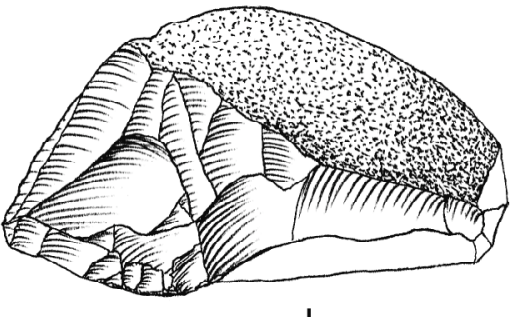

I

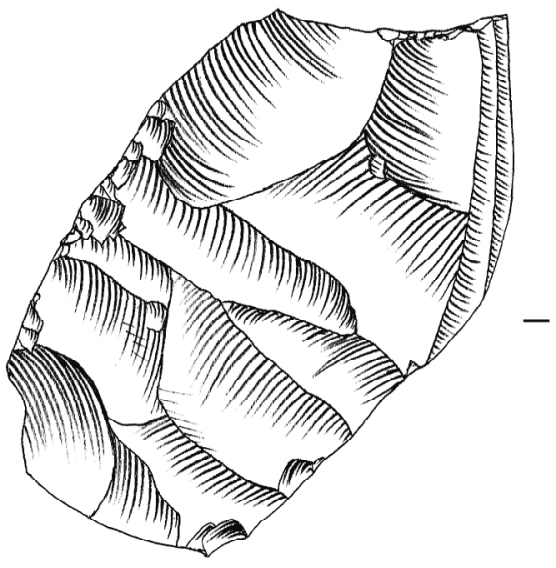

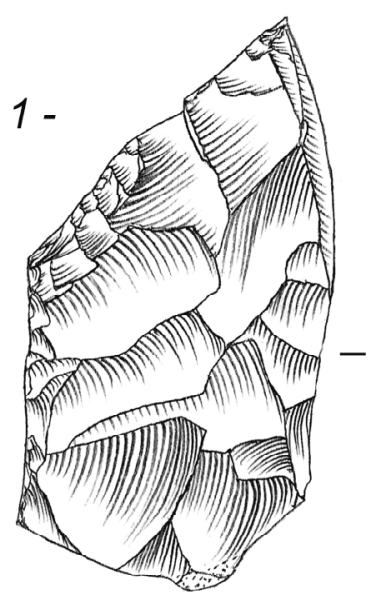
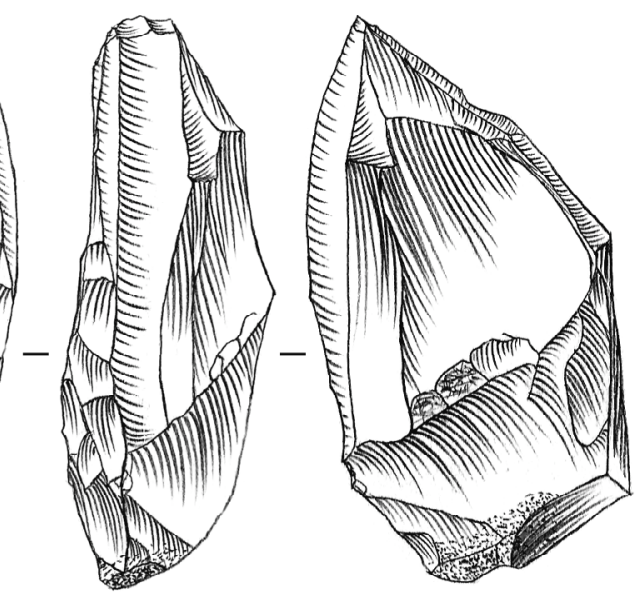

$-2$

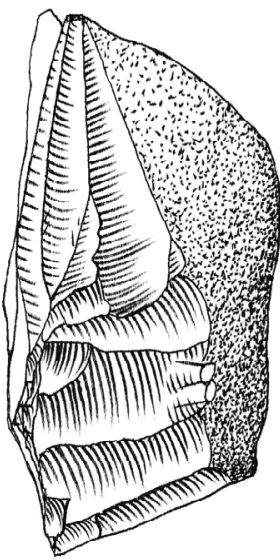

Figure 3 - Burins des Vachons, Abri 2, couche 2 (dessins D. Pesesse).

Figure 3 - Burins des Vachons, shelter 2, layer 2 (drawings $D$. Pesesse).

volume initial, qu'il s'agisse d'éclats, d'éclats diaclasiques ou même de fines plaquettes (fig 4). Les nucléus ainsi obtenus possèdent des morphologies très distinctes. Les nucléus sur lames ou sur préformes complètes sont étroits et symétriques alors que l'exploitation d'éclats épais, fréquemment corticaux, configure le volume de manière asymétrique. Ce facteur trouve une incidence notable sur les modalités respectives de gestion du débitage.

Le positionnement de la table s'effectue dans la partie étroite du volume, transversalement à la longueur du support. Dans certains cas, l'allongement des enlèvements a été privilégié par le biais d'une orientation de la table dans le grand axe du nucléus (fig. $3, n^{\circ} 1$ ).

L'exploitation de nucléus étroits permet de maintenir la progression du débitage au centre du volume, de manière frontale, sans investir fortement les flancs (fig. 1, $n^{\circ} 1$; fig. 4). En revanche, l'asymétrie des nucléus épais implique un positionnement de la table légèrement différent car l'importante convexité du flanc cortical réduit la possibilité de déborder vers la face supérieure. L'envahissement se trouve plus fortement limité si le plan de frappe est aménagé par retouche directe. Dans ce cas, la table est alors implantée de manière désaxée entre la tranche et la face inférieure de l'éclat (fig. $1, \mathrm{n}^{\circ} 2$; fig. 2).

Le débordement vers la face inférieure s'effectue par l'intermédiaire de lamelles torses et outrepassantes, emportant un pan de la face inférieure en bordure de table ou les négatifs de la mise en forme. L'entretien de la face supérieure passe préalablement par l'extraction d'une tablette emportant la troncature (Le Brun-Ricalens et Brou 2003). Cet enlèvement burinant met en place une angulation plan de frappe/face supérieure et une nervure d'accroche pour le percuteur, favorables à l'envahissement de la face supérieure. Un éclat de cadrage peut alors être extrait à la jonction table/face supérieure. Cet éclat très standardisé présente une morphologie triangulaire, évasée en partie distale. II emporte sur son bord droit une part des négatifs lamellaires issus de la table, en son centre, le négatif du précédent éclat de même type, et en son bord gauche, un pan de la face supérieure (emportant soit les négatifs des enlèvements antérieurs, soit du cortex) (fig. $5, n^{\circ} 3$ et 4 , et pour les négatifs de ces éclats fig. $1, n^{\circ} 2$; fig. $\left.3, n^{\circ} 2\right)$. L'exploitation d'éclats épais asymétriques implique donc une gestion différentiée des flancs. La progression, dans ce cas, n'est pas véritablement semi-tournante, puisque la position des produits recherchés ne varie pas au cours de la réduction.

\section{Les produits recherchés}

L'extraction de supports de cadrage en bordure de table constitue une des spécificités de la production de type Vachons. II répond à une intention de délimiter précisé- 

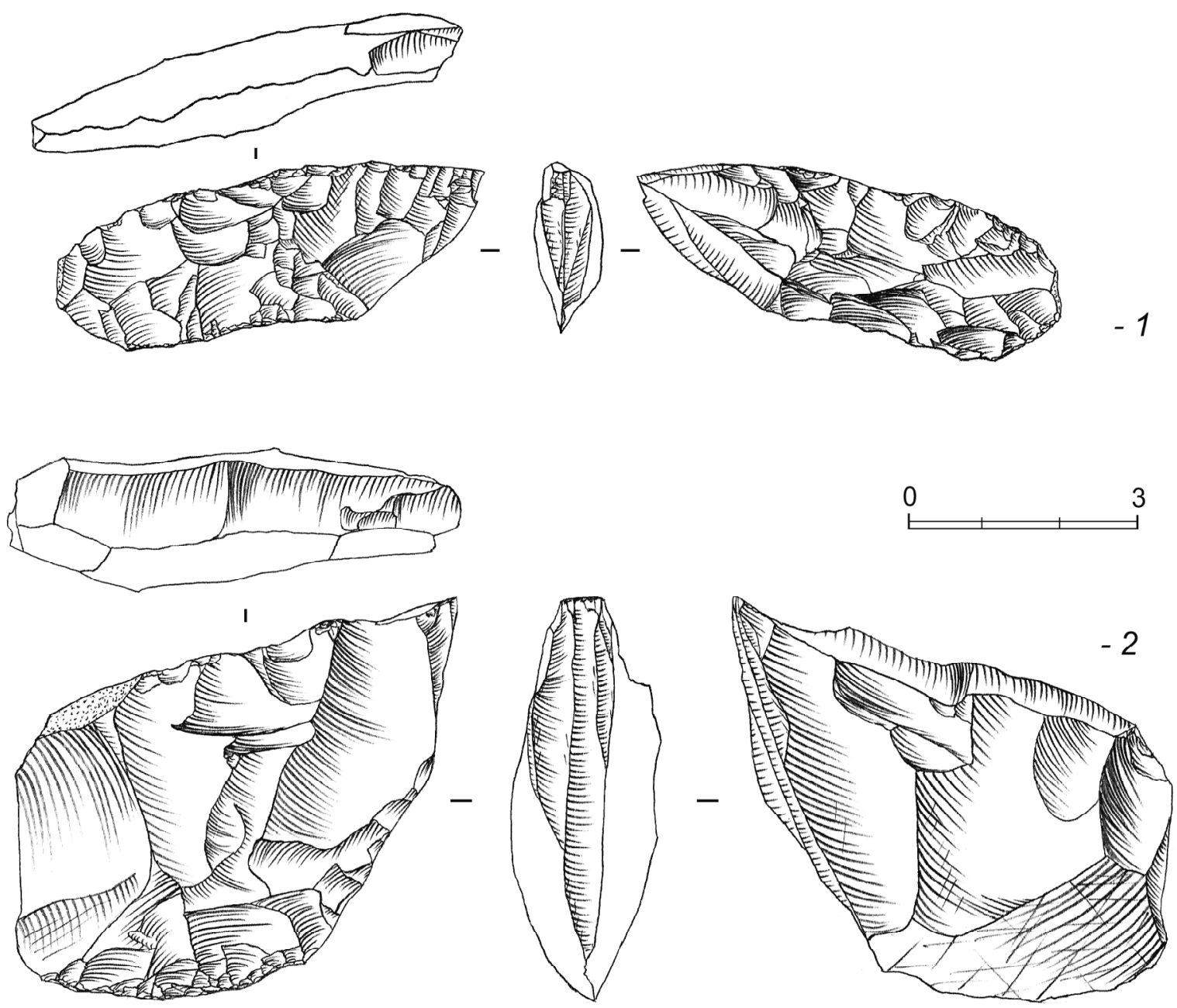

Figure 4 - Burins des Vachons, Abri 2, couche 2 (dessins D. Pesesse).

Figure 4 - Burins des Vachons, shelter 2, layer 2 (drawings D. Pesesse).

ment la table, en réduisant les convexités latérales, liées à l'exploitation d'une tranche d'éclat. Cela contribue également à configurer la délinéation latérale des enlèvements. Ainsi, la morphologie des supports lamellaires extraits au centre de la table n'est pas tributaire de la morphologie initiale du volume exploité.

Compte tenu de l'absence de fraction lamellaire dans la série étudiée, la morphologie des produits recherchés ne peut être appréhendée que par l'observation des négatifs lamellaires et la compréhension des logiques d'agencements des supports. Deux types d'enlèvements peuvent ainsi être distingués. Les supports extraits au centre de la table sont droits et rectilignes car l'onde de choc détache l'enlèvement avant d'arriver en fin de table. Les supports, extraits à partir du négatif du bord droit des précédents, donc légèrement désaxés vers la face inférieure, montrent une torsion dextrogyre ténue en partie proximale et une faible courbure. L'outrepassage des lamelles latérales et la convergence des enlèvements lamellaires confèrent une extrémité acuminée à ces deux types de produits. D'un point de vue dimensionnel, les négatifs lamellaires dessinent des gabarits très distincts, de 17 à $60 \mathrm{~mm}$ de longueur (jusquà $80 \mathrm{~mm}$ pour le plus long), et 3 à $12 \mathrm{~mm}$ de large.

\section{SYNTHÈSE}

Seules certaines orientations techniques, certaines conceptions de la production de type Vachons transparaissent dans cette collection, très fragmentaire. En dépit de ce biais, la diversité et la complexité de ce système technique se profilent clairement. La production de type Vachons ne peut être comprise comme l'expression d'une unique modalité technique mais comme un " concept " de production se déclinant selon différents axes. Cette conception se manifeste par la réalisation d'un objectif, la production de lamelles subrectilignes à rectilignes, poin- 


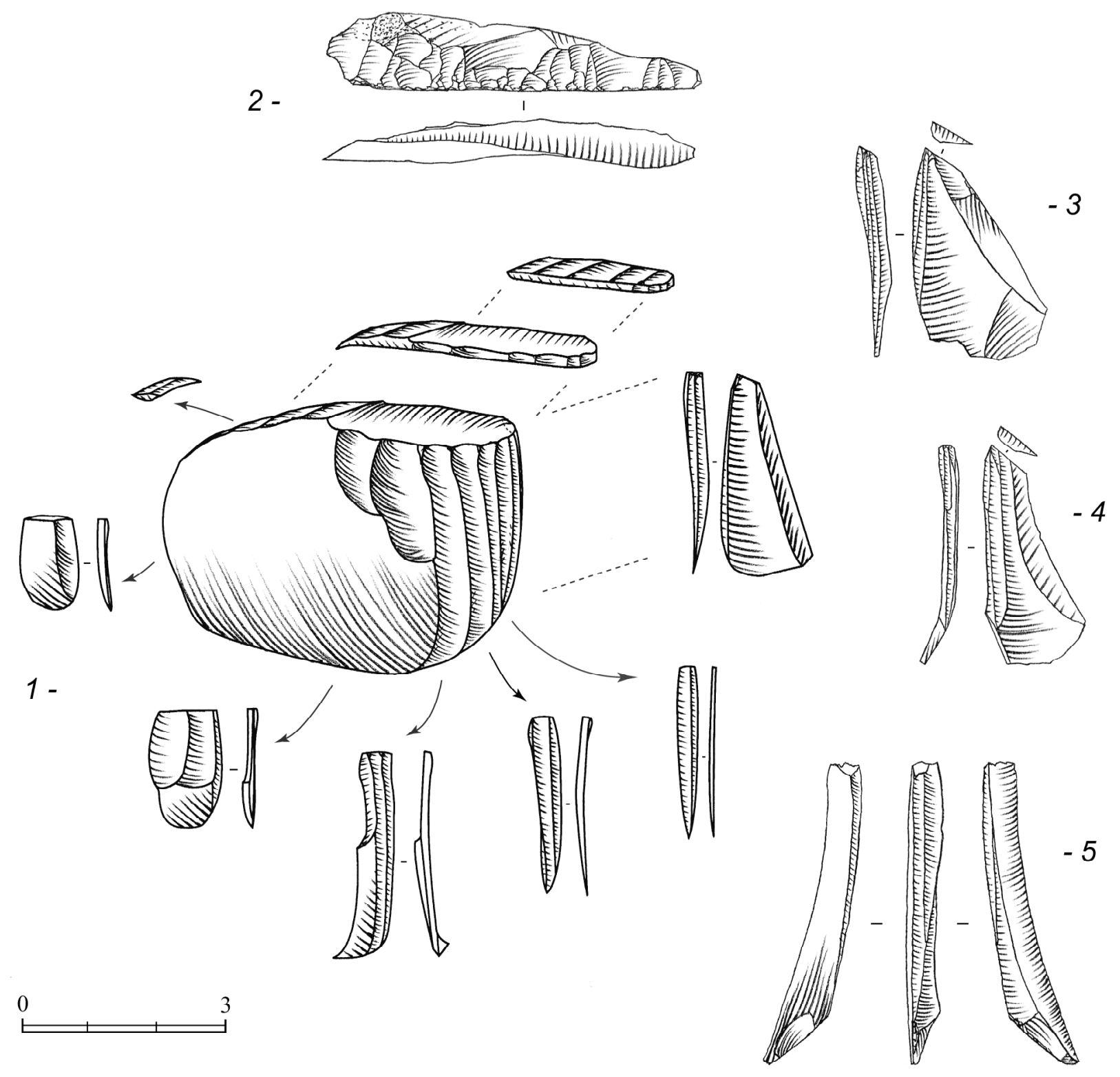

Figure 5 - Schéma de production de type Vachons sur éclat épais ( $\left.n^{\circ} 1\right)$ et sous-produits provenant de la couche 2 ( $n^{\circ} 2$ à 5$)$ (dessins D. Pesesse).

Figure 5 - Schématical process of the Vachons's production $\left(n^{\circ} 1\right)$ and sub-products from the layer 2 ( $n^{\circ} 2$ to 5$)$ (drawings D. Pesesse).

tues, selon différentes modalités techniques, liées notamment à la morphologie initiale des supports sélectionnés (fig. 5). L'apparente variabilité du burin des Vachons disparaît ainsi derrière une manière unique de concevoir la production. Seule l'étude d'ensembles lithiques homogènes sera à même de définir si à ces modalités peuvent être corrélées des différences d'objectifs notables, et ainsi de distinguer des sous-types de production.

La reconnaissance d'une logique de production de type Vachons s'avère d'autant plus importante que les débitages réalisés sur tranches d'éclats peuvent présenter de fortes convergences techniques. Le mode de ravivage des burins des Vachons s'effectue en effet selon une modalité documentée pour le Magdalénien ancien de Thèmes (Le Brun-Ricalens et Brou 2003). De même, l'entretien latéral de la table à la jonction de la face supérieure, par l'extraction d'un sous-produit très spécifique, trouve de forts points de comparaison avec les productions sur burins carénés fins de l'Aurignacien récent du Levant (Tixier 1974 ; Bergman 1987 ; Soriano 1997), mais également du Gravettien ancien de la Vigne Brun (Pesesse 2002, 2003). La reconnaissance depuis ces dix demières années de nombreux schémas opératoires basés sur l'exploitation de la tranche d'un éclat tant pour le Paléolithique supérieur (Le Brun-Ricalens et Brou à paraître ; Le Brun-Ricalens et Brou 2003 ; Le Brun-Ricalens 2005 ; Brou et Le Brun- 
Ricalens 2005) que pour certaines industries du Paléolithique moyen récent (Slimak 1999, Slimak et Lucas 2005) révèle la multiplicité des objectifs et des réponses adoptées par les artisans paléolithiques pour faire face aux contraintes volumétriques et morphologiques inhérentes à l'exploitation d'une tranche d'éclat. Si des convergences techniques apparaissent entre elles, des logiques d'exploitations différentes permettent, selon l'agencement des supports et des éléments de cadrage, d'exprimer des objectifs distincts.

Au-delà de convergences techniques, des formes de passage peuvent apparaître entre différents procédés. La série des Vachons offre des exemples de pièces partageant des caractères de gestion mixte, depuis le burin busqué à cadrage latéral, jusqu'au burin des Vachons à coche d'arrêt (fig. 6). Ces éléments tendent à nuancer sinon réduire l'impression de rupture créée par les exemplaires les plus typiques de chacun des procédés lamellaires.

\section{LES GISEMENTS}

Très tôt des comparaisons furent établies entre le niveau 2 des Vachons et des ensembles aurignaciens à fort indice de burins (Bouyssonie 1948). En 1950, J. Bouyssonie remarque que "la similitude de l'industrie du burin au niveau 2 de ces 3 gisements : Chanlat, Bouitou, Vachons est frappante. " (Bouyssonie et Delsol 1950, p 188). Ce niveau d'Aurignacien évolué fut ensuite rapproché du niveau D2S de Caminade-Est (Bouyssonie et SonnevilleBordes 1956, p. 284), bien que D. de Sonneville-Bordes ne décrive pas de burin caréné proche des Vachons dans ces niveaux riches en burins busqués (Sonneville-Bordes 1970). D. de Sonneville-Bordes compara cette industrie à l'Aurignacien II de la Ferrassie et de la Faurélie (SonnevilleBordes 1960, p. 131). En 1962, H. Delporte mentionne la présence " $d u$ " burin caréné " de l'Aurignacien évolué des Vachons " dans la couche 17 de l'Abri du Facteur (Delporte 1962), présent dans les couches 17 à 15 (Delporte 1968). La dénomination " burin des Vachons " et la définition proposées par M. Perpère (Perpère $1972 \mathrm{a}$ et b) confèrent enfin une certaine lisibilité à cette catégorie d'outils. En Charente, $M$. Perpère retrouve ces burins particuliers dans la couche B de l'Abri du Chasseur (Perpère 1975, p. 245) bien que l'homogénéité des niveaux présentant de tels témoignages de "survivance " (Balout 1956) de l'Aurignacien au Gravettien aie pu être remise en question (Perpère 1975, Sonneville-Bordes 1985). A. Morala rencontre ces burins dans l'Abri Peyrony, niveau 9a-10 (Morala 1982, 1984). Par la suite, P.-Y. Demars reconnait ces outils dans la couche supérieure de Chanlat (Demars 1982), puis à Roc de Combe, couches 5 et 6 et à Gorse (Demars et Laurent 1989 ; Demars 1994). Le Grand Abri de la Ferrassie a livré à $\mathrm{H}$. Delporte des burins "du type des Vachons " dans la partie sommitale de la séquence, à partir de la couche K3b (Delporte 1984). Dans les sériations successives de l'Aurignacien, H. Delporte ne prit pourtant jamais en compte la présence de burins des Vachons, comme un élément de subdivision des phases récentes de l'Aurignacien (Delporte 1962, 1968, 1984, 1991). Les burins des Vachons sont aussi présents dans la couche VIII du Flageolet I et dans la couche 5 (peut-être dans les couches
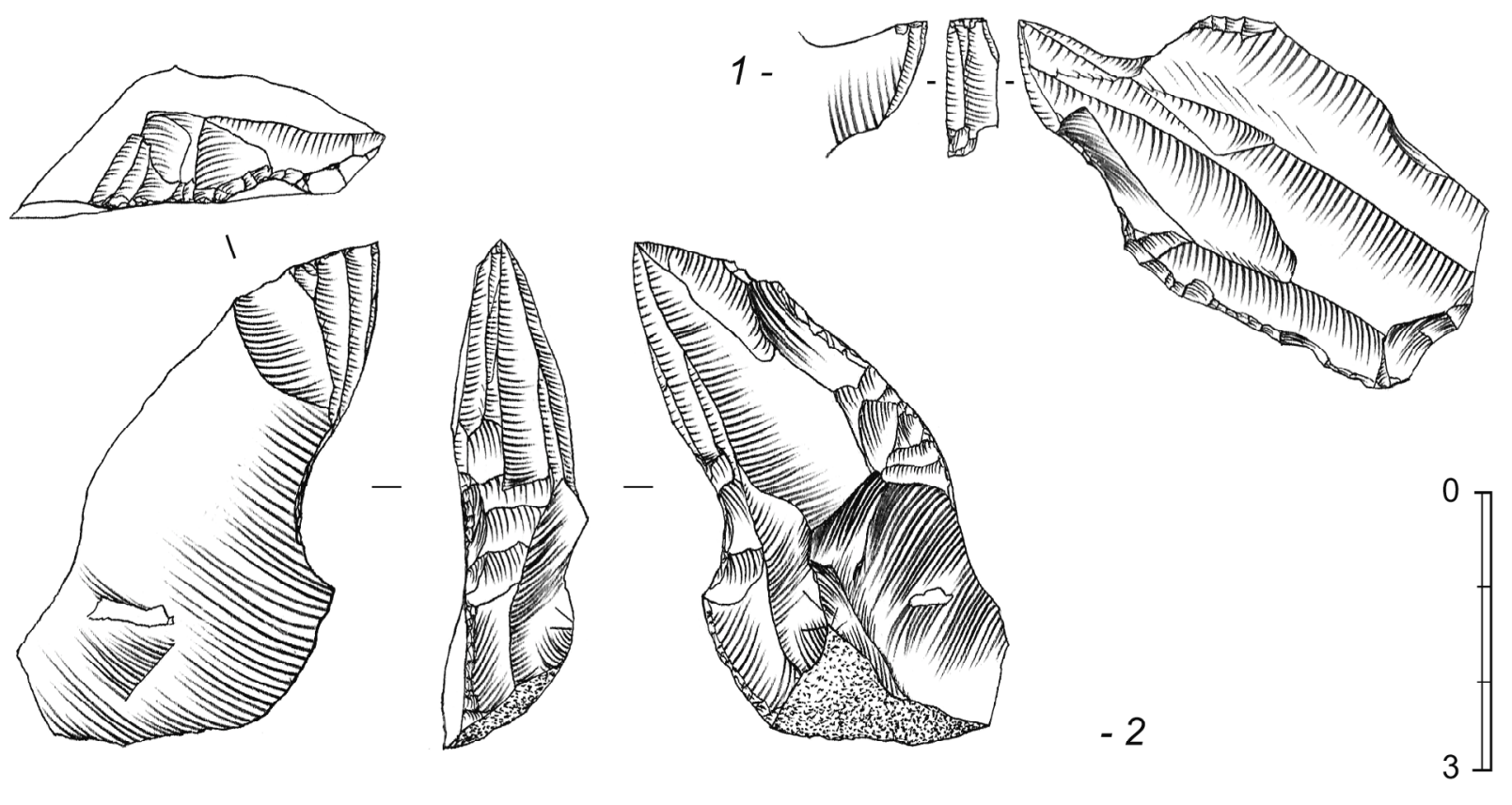

Figure 6 - Burin busqué, $n^{\circ} 1$ : Abri 2, couche 2, et burin busqué à tendance Vachons, $n^{\circ} 2$ : Abri 1, couche 2 (dessins D. Pesesse).

Figure 6 - Burin busqué, $n^{\circ} 1$ : shelter 2 , layer 2 and burin busqué near of the Vachon’s type, $n^{\circ} 2$ : shelter 1 , layer 2 (drawings D. Pesesse). 
4 et 6) de la grotte Maldidier (Rigaud 1982). L'importante séquence de l'Abri Pataud abonde en ce sens, les burins des Vachons apparaissent dans la partie supérieure, à partir de la couche 7 (Chiotti 2003).

En résumé, les burins des Vachons furent toujours rencontrés en sommet de séquence aurignacienne, dans des niveaux sus-jacents à des assemblages à grattoirs à museaux, et associés à des burins busqués (fig. 7). Ces éléments de stratigraphie confirment leur valeur chrono-culturelle reconnue par P.-Y. Demars (Demars 1994 ; Demars et Laurent 1989) comme marqueur de la fin de l'Aurignacien dans les gisements du Nord de l'Aquitaine et des Charentes.

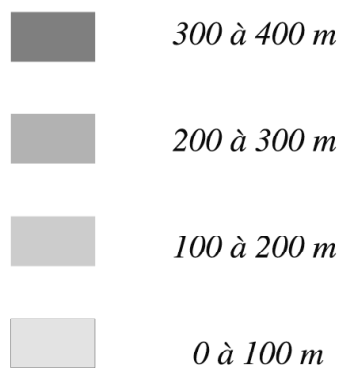

0 50 $\mathrm{km}$
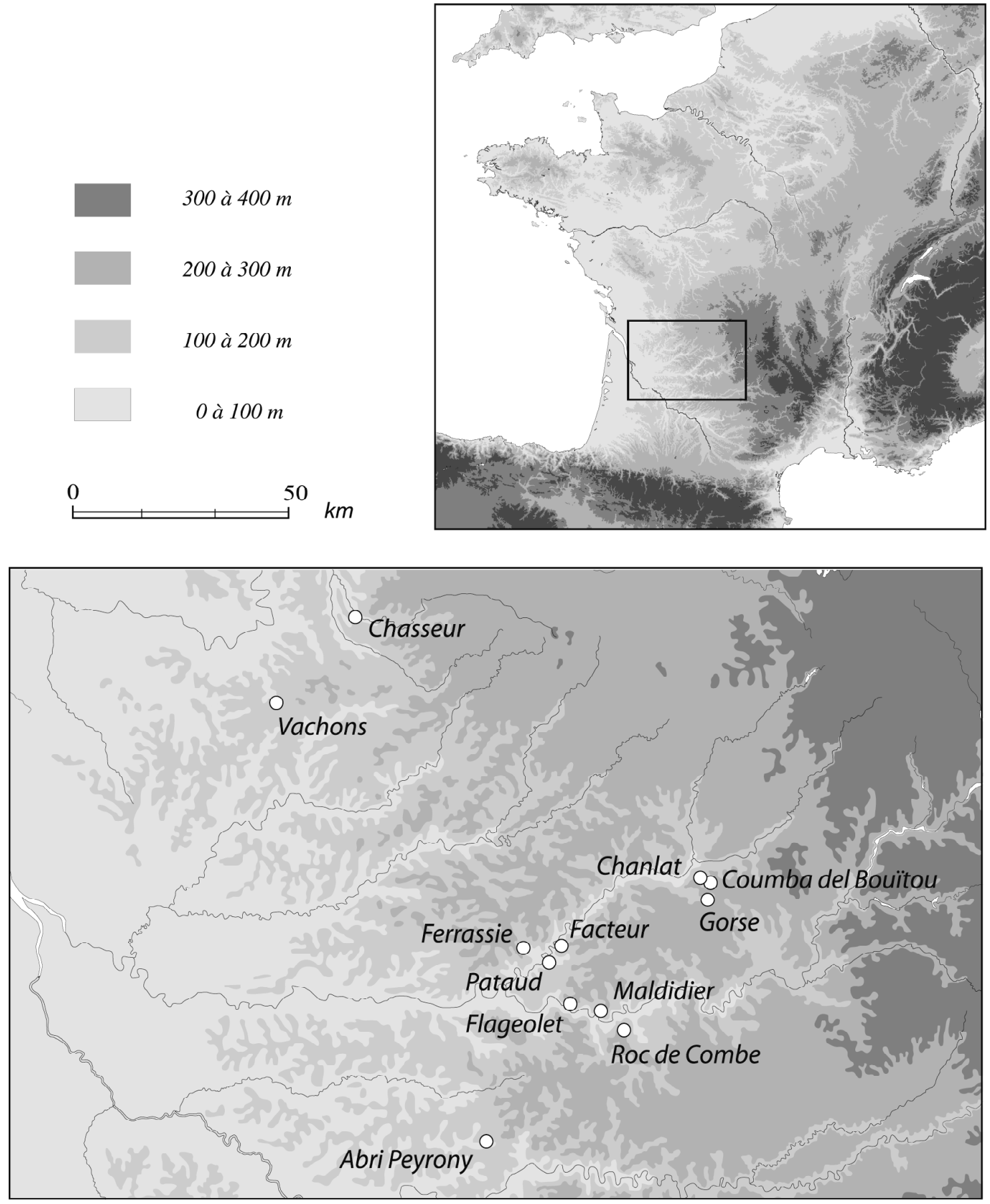

Figure 7 - Répartition des gisements à burins des Vachons cités dans le texte.

Figure 7 - Localisation of the sites including burins des Vachons called in the text. 

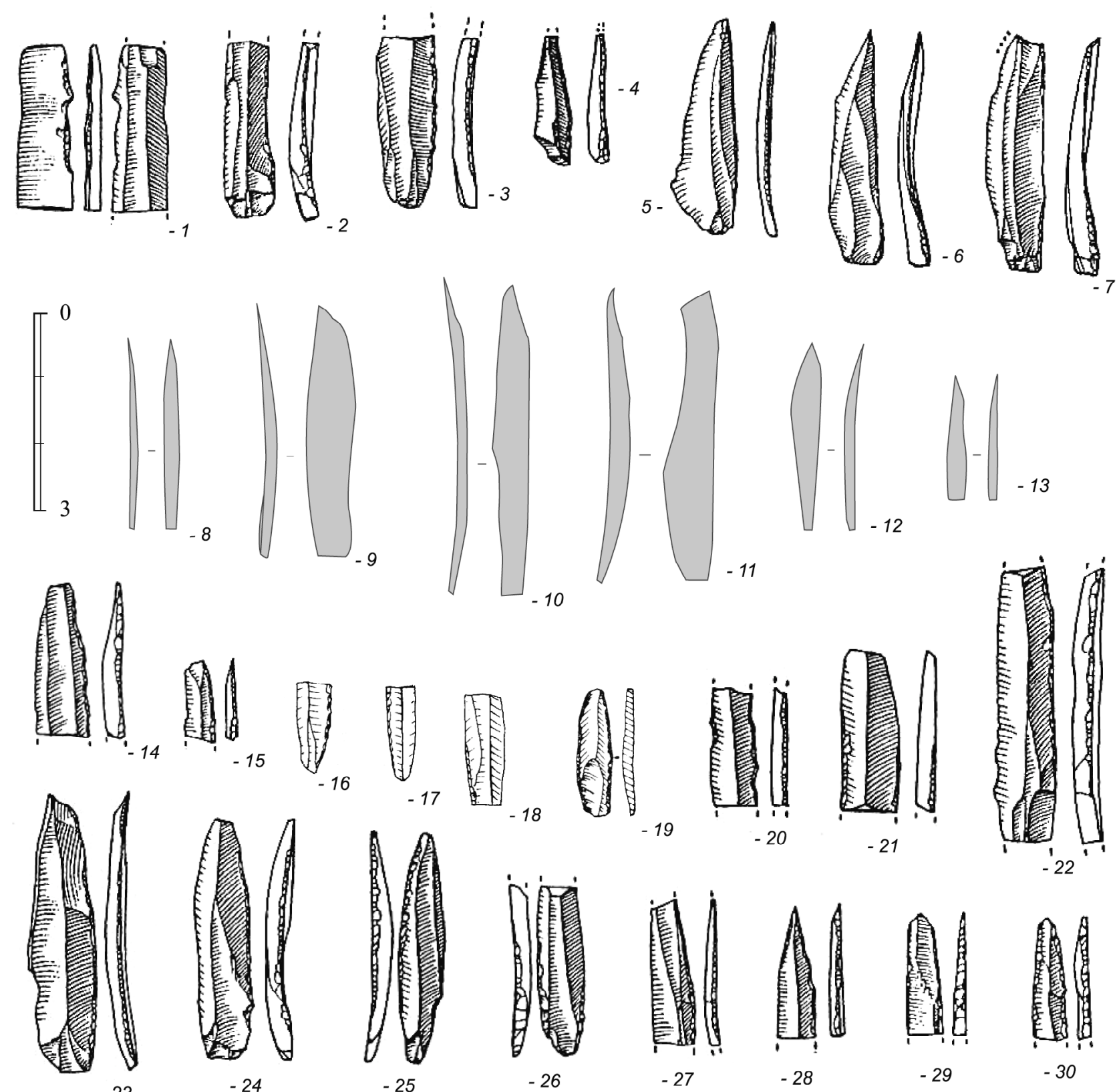

23

$-25$

$-27$

$-28$

$-29$

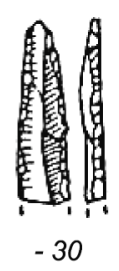

Figure 8 - Négatifs et profils des derniers enlèvements de plein débitage des burins des Vachons (8 à 13), et lamelles à retouche directe marginales du Flageolet I, (couche VIII : 1 à 7), la Ferrassie (couche I2 : 16, couche $H: 17,18$ ), l'Abri Peyrony (niveau 9a-10 : 19), et Maldidier (niveau 4 : 20, 21, 22, niveau $5: 14,23$ à 30, niveau $6: 15$ ) (dessins J.-G. Marcillaud : 1 à 7 , 14, 15, 20 à 30 ; H. Delporte : 16 à 18 ; A. Morala : 19).

Figure 8 - Negatives and profils of the last bladelets produced by burins des Vachons (8 à 13), from Flageolet l, (couche VIII : 1-7), Ferrassie (layer 12 : 16, couche $H: 17,18$ ), Abri Peyrony (layer 9a-10 : 19), and Maldidier (layer $4: 20,21,22$, layer 5 : 14, 23-30, layer 6 : 15) (drawings J.-G. Marcillaud : 1-7, 14, 15, 20-30 ; H. Delporte : 16-18 ; A. Morala : 19). 

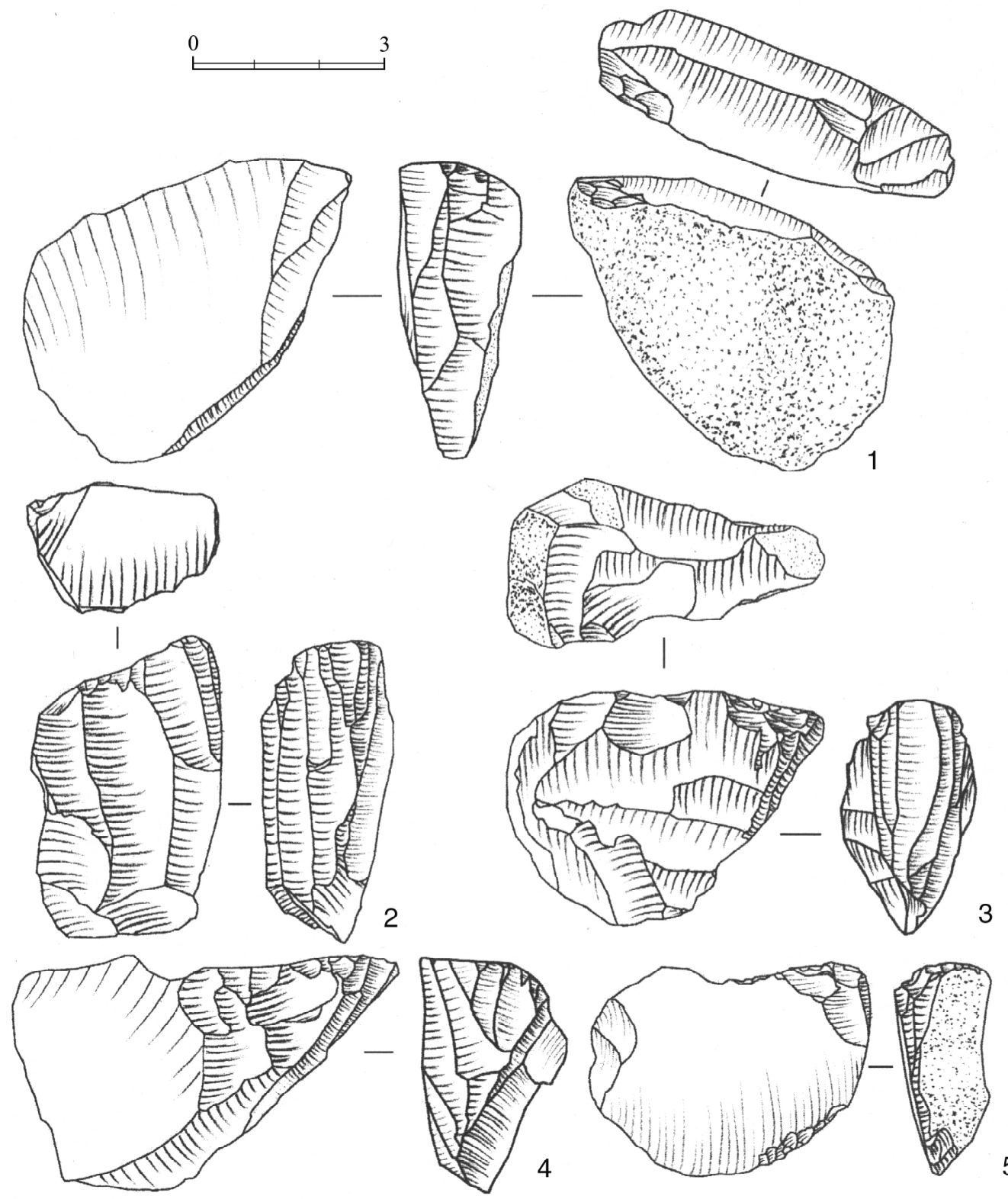

5

Figure 9 - Burins des Vachons de la Ferrassie, $n^{\circ} 1,2,4$ et 5 - couche $H^{\prime}$; $n^{\circ} 3$ - couche $H^{\prime \prime}$ (dessins A. Michel).

Figure 9 - Burins des vachons from Ferrassie $n^{\circ} 1,2,4$ et 5 - couche $H^{\prime}$; $n^{\circ} 3$ - couche $H^{\prime \prime}$ (drawings A. Michel).

\section{Les lamelles retouchées \\ associées au burin des Vachons}

Dans certains niveaux archéologiques comprenant des burins des Vachons, plusieurs populations de lamelles retouchées peuvent être distinguées. II s'agit notamment de lamelles Dufour torses dextrogyres, à retouche dextre inverse ou alterne, correspondant au sous-type Roc-deCombe (Demars et Laurent 1989). Ces supports sont produits à partir de grattoirs à museaux et de burins busqués
(Lucas 1997, 2000 ; Chiotti 1999, 2000, 2003 ; Bordes 2005). La seconde population, représentée par les lamelles Caminade, est réalisée sur des lamelles rectilignes, de très petites dimensions, portant une retouche directe sur un bord, ou, plus rarement, sur les deux (Bordes et Lenoble 2002). Ces lamelles sont issues, comme les lamelles Rocde-Combe, de burins busqués.

À côté de ces deux populations, certains supports se différencient par un module plus important, un profil rectiligne ou légèrement courbe, et par la présence d'une fine retouche 

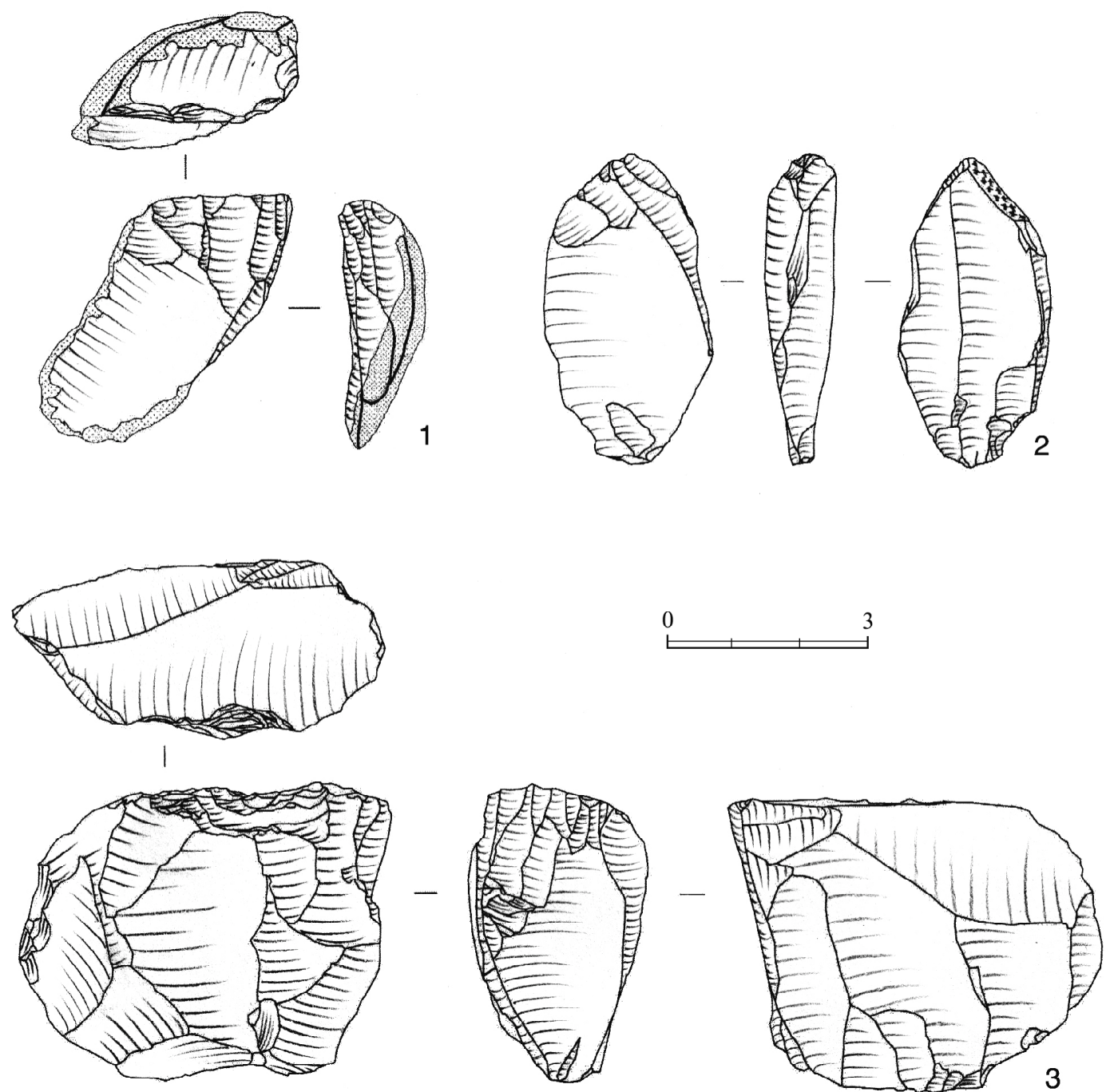

Figure 10 - Burins des Vachons du Roc-de-Combe, $n^{\circ} 1$ - couche 5; $n^{\circ} 2$ et 3 - couche 6 (dessins A. Michel).

Figure 10 - Burins des Vachons from Roc-de-Combe, $n^{\circ} 1$ - layer 5; $n^{\circ} 2$ et 3 -layer 6 (drawings A. Michel).

dextre directe. Ces lamelles retouchées s'observent notamment à l'Abri Peyrony (Morala 1984), à Maldidier, au Flageolet I (Rigaud 1982) et à la Ferrassie (Delporte 1984) (fig. 8). Ces supports présentent des caractéristiques dimensionnelles et morpho-techniques très distinctes des lamelles Dufour et Caminade de l'Aurignacien récent. En revanche, elles partagent le module et la rectitude des négatifs décrits pour la production de type Vachons (fig. $8, n^{\circ} 8$ à 13). Compte tenu de cette correspondance en termes d'objectifs, il est alors envisageable que ces lamelles à retouche directe marginale correspondent aux lamelles produites à partir des burins des Vachons. La réalisation de remontages et la discrimination d'autres schémas lamellaires concordants dans ces niveaux seront nécessaires pour vérifier pleinement cette hypothèse. L'association du burin des Vachons avec d'autres modes de productions lamellaires, grattoirs à museaux et burins busqués, pose certains questionnements concernant la stricte co-existence de ces modalités que seules de nouvelles fouilles, notamment de sites de plein-air, permettront de vérifier.

\section{Les burins des Vachons à la Ferrassie, au Roc-de-Combe et au Flageolet I}

La présence du burin des Vachons a pu être confirmée lors de l'examen du matériel de certains gisements nord-aquitains. Dans la collection Peyrony de l'Abri de la Ferrassie, conservée au Musée National de Préhistoire, ces burins se rencontrent de manière épisodique dès la couche $\mathrm{H}$ (fig. 9), attribuée à l'Aurignacien II (Peyrony 1933), leur fréquence augmente de manière significative dans les couches susjacentes, $\mathrm{H}^{\prime}$, attribuée à l'Aurignacien III, et $\mathrm{H}^{\prime \prime}$ à l'Aurignacien IV (Peyrony 1933). Pour I'heure, la position 


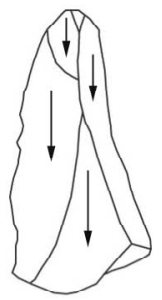

1

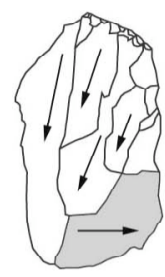

4

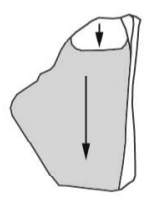

6
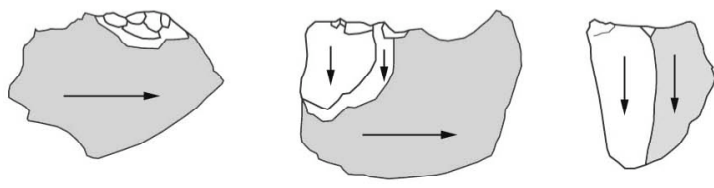

12

10

11

8

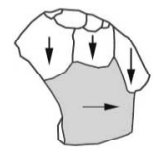

stratigraphique des burins des Vachons de la couche $\mathrm{H}$ reste discutable. Les couches 6 et 5 du Roc-de-Combe, respectivement attribuées à un Aurignacien Il et évolué par D. de Sonneville-Bordes (Sonneville-Bordes 2002) et la couche VIII du Flageolet I, décrite comme un Aurignacien récent (Lucas 2000, Rigaud 1982) recèlent effectivement ces burins spécifiques (fig. 10).

Dans ces gisements, les burins des Vachons révèlent une logique opératoire identique à celle observée dans la série éponyme. Le choix des supports de nucléus s'avère aussi varié. Toutefois, bien que certaines pièces aient été fortement investies, la mise en forme demeure généralement sommaire. Un examen de la fraction fine des séries du Roc-de-Combe et du Flageolet I a permis de retrouver les éclats de mise en forme (notamment des éclats kombéwa) et des éléments d'entretien des nucléus (fig. 11). Leurs caractéristiques techniques viennent confirmer les tendances décrites précédemment. Certains éclats kombéwa ont pu être remontés sur des burins des Vachons, dans la couche VIII du Flageolet I, mais malheureusement aucune lamelle retouchée.

La variation des types de produits, pressentie lors de l'étu-
円

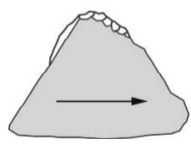

9

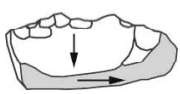

13
Figure 11 - Sous-produits de burins des Vachons du Roc-de-Combe, couche 5 $(1,2,4$ à 9) et du Flageolet I, couche 8 (3, 10 à 13) (dessins A. Michel).

Figure 11 - Sub-products of burins des Vachons from Roc-de-Combe, layer 5 (1, 2, 4 à 9) and Flageolet I, layer VIII (3, 10 à 13) (drawings $A$. Michel). de des burins-nucléus, se retrouve dans la fraction lamellaire de la couche VIII du Flageolet I, et dans une moindre mesure dans celles des couches 6 et 5 du Roc-de-Combe. Les produits recherchés étant soit tors en partie proximale, soit totalement rectilignes. Malgré cette différence, ils présentent une retouche directe sur un bord, voire les deux, affectant surtout la partie distale, renforçant ainsi l'aspect appointé de ces lamelles.

\section{BILAN ET PERSPECTIVES}

La série éponyme permet de reconsidérer la nature du burin des Vachons et de poser, en complément aux observations antérieures, des bases nouvelles pour sa définition et sa compréhension. Cette pièce peut dorénavant être considérée comme un nucléus à lamelles et non plus comme un simple outil, aspect déjà évoqué par L. Chiotti (2003). Le burin des Vachons ne correspond pas seulement à un schéma de débitage original réalisé à partir d'un burin caréné mais à un concept de production lamellaire décliné sous des 
formules très diverses, du burin caréné fin à la préforme complète. Ainsi, l'envahissement de la face inférieure ne constitue qu'un des éléments de définition et de reconnaissance du burin des Vachons et ne peut être retenu isolément comme diagnostique.

Mais l'intérêt ne réside pas dans le degré d'élaboration ou de complexité du système technique ; des productions lamellaires très normées, très investies existent durant tout l'Aurignacien. En revanche, la manière dont les objectifs de production lamellaire évoluent à partir des traditions techniques de l'Aurignacien récent constitue un élément remarquable. Après l'évolution morphologique et technologique progressive de la lamelle Dufour depuis l'Aurignacien ancien jusqu'au sous-type Roc-de-Combe, une modification importante apparaît enfin dans ce continuum. Les objectifs lamellaires, depuis le processus de production de type burin busqué, dont la rigidité opératoire assure la constance du profil et la faible dispersion dimensionnelle des produits recherchés, les nano-lamelles torses (Lucas 1997, 2000 ; Chiotti 1999, 2003 ; Chazan 2001 ; Bordes 2005 ; Bordes et Lenoble 2002), évoluent en faveur d'un élargissement dimensionnel de la gamme des produits et une modification notoire des paramètres. Les lamelles provenant du burin des Vachons mesurent en effet de 20 à $60 \mathrm{~mm}$ de longueur, possèdent un profil rectiligne à subrectiligne et une extrémité distale aigüe. La généralisation de la retouche directe, apparue avec la lamelle Caminade, constitue un des paramètres de cette évolution.

Toutefois, bien que les logiques opératoires des burins busqués et des burins des Vachons diffèrent notablement, une mise en opposition stricte de ces processus ne permettrait pas d'appréhender les mécanismes d'évolution de ces systèmes techniques. Dans ce sens, le burin des Vachons pourrait être abordé comme le prolongement technique de certaines innovations manifestées, dès la phase à burins busqués, avec la lamelle Caminade (Bordes et Lenoble 2002). En effet, cet outil cristallise déjà certaines intentions, dont la rectitude, qui seront les éléments constitutifs de la production de type Vachons.

La reconnaissance d'un tel objectif dans les phases récentes de l'Aurignacien nord-aquitain modifie profondé ment notre compréhension des demières expressions de cette culture. Au-delà des variations techniques, le burin des Vachons révèle une modification profonde dans la manière même de concevoir la production dans l'histoire lithique aurignacienne. Ce changement conceptuel permet de mieux envisager les innovations et les restructurations ultérieures que ne laissait entrevoir la rigidité du processus de production de type burin busqué.

Un important travail reste à accomplir dans la compréhension de l'Aurignacien récent, notamment dans la caractérisation des objectifs de production des burins des Vachons, dans la documentation de la diversité de ce système technique, dans la définition de son ancrage dans la tradition technique et économique aurignacienne. La manière dont les productions lamellaires évoluent à partir de l'Aurignacien récent, dans les objectifs et leur intégration à la sphère laminaire (Bordes et Lenoble 2002 ; Michel 2005) constitue une voie d'investigation privilégiée à la compréhension des sociétés de la fin de l'Aurignacien. Cette contribution ne constitue qu'un jalon de cette enquête.

\section{Remerciements}

Nous tenons tout d'abord à remercier Jean-Jacques Cleyet-Merle et l'ensemble du personnel du Musée National de Préhistoire. Nos remerciements s'adressent aussi à J.-P. Rigaud qui nous permet d'étudier les industries aurignaciennes du Flageolet I, et à J. Airvaux qui nous a offert l'occasion de voir la série des Vachons conservée à Poitiers. Enfin, Jean-Guillaume Bordes, François Bon et Foni Le Brun-Ricalens méritent une fois de plus une sincère révérence.

\section{BIBLIOGRAPHIE}

BALOUT L. 1956 - L'Abri du Chasseur au Bois du Roc, commune de Vilhonneur (Charente), Note préliminaire, Congrès Préhistorique de France, $\mathrm{XV}^{\circ}$ session, PoitiersAngoulême, p. 199-205.

BERGMAN C. A. 1987 - Ksar Akil, Lebanon, A technologi$\mathrm{cal}$ and typological analysis of the later paleolithic levels of Ksar Akil, vol II : levels XII-VII, with contribution of L. Copeland and M. Newcomer, Oxford, British Archeological Repports, Series 329.

BORDES J.-G. 2005 - La séquence aurignacienne du Nord de l'Aquitaine : variabilité des productions lamellaires à Caminade-Est, Roc-de-Combe, le Piage et CorbiacVignoble II, In : Le Brun-Ricalens F. (Ed.), Productions lamellaires attribuées à l'Aurignacien : Chaînes opératoires et perspectives techno-culturelles. XIV ${ }^{\circ}$ congrès de I'UISPP, Liège (2-8 septembre 2001). Luxembourg : ArchéoLogiques $\mathrm{n}^{\circ} 1$, p. 123-154.

BORDES J.-G., LENOBLE A. 2002 - La " lamelle Caminade" : un nouvel outil lithique aurignacien ?, Bulletin de la Société Préhistorique Française, t. 99, n4, p. 735-749.

BOUYSSONIE J. 1948 - Un gisement aurignacien et périgordien, les Vachons (Charente), L'Anthropologie, t. 52, p. 1-42.

BOUYSSONIE J., DELSOL H. 1950 - La Grotte de Chanlat près Brive (Corrèze), Congrès Préhistorique de France, XIllème session, Paris, p. 183-190.

BOUYSSONIE J., SONNEVILLE-BORDES D. 1956 - L'Abri $\mathrm{n}^{\circ} 2$ des Vachons, gisement aurignacien et périgordien, commune de Voulgézac (Charente), Congrès Préhistorique de France, XVème session, Poitiers-Angouléme, p. 271-309.

BREZILLON M. 1968 - La dénomination des objets de pierre taillée. Matériaux pour un vocabulaire des préhistoriens de langue française, IVº suppl. à Gallia Préhistoire, 417 p.

BROU L., LE BRUN-RICALENS F. 2005 - Productions lamellaires et technocomplexes paléolithiques. Incidences : le Paléolithique supérieur revisité, In : Le BrunRicalens F. (Ed.), Productions lamellaires attribuées à l'Aurignacien : Chaînes opératoires et perspectives technoculturelles. XIV ${ }^{\circ}$ congrès de l'UISPP, Liège (2-8 septembre 2001). Luxembourg : ArchéoLogiques n¹, p. 123-154. 
CHAZAN M. 2001 - Bladelet production in the Aurignacien of la Ferrassie (Dordogne, France, Lithic Technology, 26/1, p. 16-28.

CHIOTTI L. 1999 - Les industries lithiques de l'Aurignacien de l'Abri Pataud, les Eyzies-de-Tayac (Dordogne) : étude technologique et typologique, Mémoire de Doctorat, Museum d'Histoire Naturelle, Institut de Paléontologie Humaine, Paris.

CHIOTTI L. 2000 - Lamelles Dufour et grattoirs aurignaciens (carénés et à museaux) de la couche 8 de l'Abri Pataud (Les Eyzies-de-Tayac, Dordogne), L'Anthropologie, t. $104, n^{\circ} 2$, p. 239-263.

CHIOTTI L. 2003 - Les productions lamellaires dans l'Aurignacien de l'Abri Pataud, Les Eyzies-de-Tayac (Dordogne), Gallia Préhistoire, t. 45, p. 113-156.

COIFFARD J. 1914 - Station des Vachons, commune de Voulgézac, Congrès de l'Association Française pour l'Avancement des Sciences, Havre, p. 623-627.

COIFFARD J. 1922 - Station des Vachons, Abri 2, Congrès de l'Association Française pour l'Avancement des Sciences, Montpellier, p. 492.

DELPORTE H. 1962 - Les niveaux aurignaciens de l'Abri du Facteur, à Tursac et l'évolution générale de l'Aurignacien en Périgord, Bulletin de la Société d'Etudes et de Recherches Préhistoriques, t. 11, p. 107-126.

DELPORTE H. 1968 - L'Abri du Facteur à Tursac, Gallia Préhistoire, t. 11, p. 1-112.

DELPORTE H. 1984 - L'Aurignacien de La Ferrassie, in Le Grand Abri de la Ferrassie. Fouilles 1968-1973. Etudes Quaternaires, mémoire $\mathrm{n}^{\circ} 7$, Institut de Paléontologie Humaine, p. 145-234.

DELPORTE H. 1991 - La séquence aurignacienne et périgordienne sur la base des travaux récents réalisés en Périgord, Bulletin de la Société Préhistorique Française, t. $88, n^{\circ} 8$, p. $248-256$.

DEMARS P.-Y. 1982 - Les grattoirs carénés et à museau, les burins busqués et carénés, les pièces nucléiformes dans le bassin de Brive, Approche stylistique, Bulletin de la Société Préhistorique Française, t. 79, p. 341-368.

DEMARS P.-Y. 1994 - L'économie du silex au Paléolithique supérieur dans le Nord de l'Aquitaine, Thèse d'Etat, Université de Bordeaux I, 2 vol.

DEMARS P.-Y., LAURENT P. 1989 - Types d'outils lithiques du Paléolithique supérieur en Europe, Cahiers du Quatemaire, 14, Paris, Presses du CNRS.

FONTAINE A. 2000 - Etude d'une ancienne collection du Gravettien, Site des Vachons (Voulgézac, Charente), Bulletin de la Société Préhistorique Française, t. 97, n², p. 191-198.
HEINZELIN DE BREAUCOURT J. de 1962 - Manuel de typologie des industries lithiques, Bruxelles, $74 \mathrm{p}$.

LE BRUN-RICALENS F. 2005 - Chronique d'une reconnaissance attendue. Outils "carénés ", outils " nucléiformes ": nucléus à lamelles. Bilan après un siècle de recherches typologiques, technologiques et tracéologiques, In : Le Brun-Ricalens F. (Ed.), Productions lamellaires attribuées à l'Aurignacien : Chaînes opératoires et perspectives techno-culturelles. $\mathrm{XIV}^{\circ}$ congrès de I'UISPP, Liège (2-8 septembre 2001). Luxembourg : ArchéoLogiques $n^{\circ} 1$, p. 123-154.

LE BRUN-RICALENS F., BROU L. 2003 - Burins carénés-nucléus à lamelles : identification d'une chaîne opératoire particulière à Thèmes (Yonne) et implications, Bulletin de la Société Préhistorique Française, tome 100 , p. $67-83$.

LE BRUN-RICALENS F., BROU L. à paraître - Burins carénés-nucléus à lamelles : identification d'une chaîne opératoire particulière à Thèmes (Yonne) et implications, in : Fosse G. (dir), Le Paléolithique supérieur et le Mésolithique dans le Nord-Est de la France et les pays limitrophes, Actes de la table ronde de Valenciennes, Valenciennes 18-19 octobre 1997, Cahiers d'Archéologie du Nord.

LUCAS G. 1997 - Les lamelles Dufour du Flageolet I (Bézenac, Dordogne) dans le contexte aurignacien, Paléo, $n^{\circ} 9$, p. 191-219.

LUCAS G. 2000 - Les industries lithiques du Flageolet I (Dordogne) : approche économique, technologique, fonctionnelle et analyse spatiale, Mémoire de Doctorat, Université de Bordeaux I, 2 vol., 603 p.

MICHEL A. 2005 - L'Aurignacien récent en Aquitaine : bilan documentaire et étude du débitage laminaire du niveau D2S de Caminade Est (La Canéda, Dordogne), Mémoire de Master 2, Université de Bordeaux 1, 75p.

MORALA A. 1982 - Abri Peyrony, Gallia Préhistoire, t. 25, fasc. 2, p. 421-423.

MORALA A. 1984 - Abri Peyrony, Gallia Préhistoire, t. 27, fasc. 2, p. 297-298.

PERPERE M. 1972a - Remarques sur l'Aurignacien en Poitou-Charentes, L'Anthropologie, t.76, p. 387-425.

PERPERE M. 1972b - Les burins aurignaciens du gisemant des Vachons (Charente), Congrès préhistorique de France, XIXème session, Auvergne, 1969, p. 320-323.

PERPERE M. 1975 - Grands gisements aurignaciens de Charente, L'Anthropologie, t. 79, p. 243-276.

PERPERE M. 1977 - L'industrie des Vachons et l'Aurignacien en Poitou-Charentes, L'Anthropologie, t. 81, p. 377-410. 
PESESSE D. 2002 - Les burins de l'unité OP10 de la Vigne Brun (Loire), Caractérisation technologique de la variabilité typologique, mémoire de Maîtrise, Université Aix-Marseille I.

PESESSE D. 2003 - Approche du comportement technique au Gravettien : l'industrie lithique de l'unité OP10 de la Vigne Brun, mémoire de DEA, Université Aix-Marseille I.

PEYRONY D. 1933 - Les industries «Aurignaciennes» dans le bassin de la Vézère. Bulletin de la Société Préhistorique Française.t. 30, p.543-559

RIGAUD J.-P. 1982 - Le Paléolithique en Périgord : les données du sud-ouest sarladais et leurs implications, Thèse de Doctorat de I'Université de Bordeaux I, 2 tomes.

SLIMAK L. 1999 - Mise en évidence d'une composante laminaire et lamellaire dans un complexe moustérien du sud de la France, Paléo, n¹1, p. 89-109.

SLIMAK L. et LUCAS G. 2005 - Le débitage lamellaire, une invention aurignacienne ? In : Le Brun-Ricalens F. (Ed.), Productions lamellaires attribuées à l'Aurignacien : Chaînes opératoires et perspectives techno-culturelles. XIV congrès de I'UISPP, Liège (2-8 septembre 2001). Luxembourg : ArchéoLogiques n¹, p. 123-154.

SONNEVILLE-BORDES D. de 1960 - Le Paléolithique supérieur en Périgord. Imprimeries Delmas, Bordeaux, $558 \mathrm{p}$.
SONNEVILLE-BORDES D. de 1970 - Les industries aurignaciennes de l'Abri de Caminade-Est, commune de la Canéda (Dordogne), Quaternaria, XIII, Roma, p. 9-43.

SONNEVILLE-BORDES D. de 1985 - Variabilités typologiques dans les outillages lithiques. Remarques sur leurs significations au Paléolithique supérieur, in : La signification culturelle des industries lithiques, M. Otte éds, Actes du colloque de Liège du 3 au 7 septembre 1984, Studia Praehistorica Belgica, 4, BAR International Series, 239, p. 391-419.

SONNEVILLE-BORDES D. de 2002 - Les industries du Roc-de-Combe (Lot). Périgordien et Aurignacien. Préhistoiredu Sud-Ouest. 9, fascicule 2, p. 121-161.

SONNEVILLE-BORDES D., PERROT J. 1956 - Lexique typologique du Paléolithique supérieur, Outillage lithique : IV Burins, Bulletin de la Société Préhistorique française, tome $53, n^{\circ} 8$, p. $408-412$.

SORIANO S. 1997 - La production de lamelles torses dans les niveaux du Paléolithique supérieur ancien d'Umm El Tlel, Préhistoire d'Anatolie, Genèse de deux mondes, Liège, ERAUL 85, p. 731-748.

TIXIER J. 1974 - Ksar'Akil, Liban (1969-1974), Paleorient, 2., p. $183-185$ 
